\title{
Demonstration of a cavity-enhanced optical parametric chirped-pulse amplification system
}

\author{
Aleem Siddiqui,* Kyung-Han Hong, Jeffrey Moses, Jian Chen, F. Ömer Ilday, and Franz X. Kärtner \\ Department of Electrical Engineering and Computer Science and Research Laboratory of Electronics, \\ Massachusetts Institute of Technology (MIT), Cambridge, Massachusetts 02139, USA \\ ${ }^{*}$ Corresponding author: siddiq@mit.edu
}

Received January 11, 2011; accepted February 10, 2011;

posted February 25, 2011 (Doc. ID 140828); published March 28, 2011

\begin{abstract}
The use of a low finesse enhancement cavity resonant with a low average power $(<1 \mathrm{~W})$ and a high repetition rate ( $78 \mathrm{MHz}$ ) pump source is shown to achieve $55 \%$ conversion efficiency into signal and idler from the coupled pump in an optical parametric process, whereas an equivalent amount of pump power in a single-pass configuration leads to negligible conversion. Careful comparison of the intracavity conversion process to the single-pass case is performed to assess the underlying impedance matching that yields the high conversion results. (C) 2011 Optical Society of America
\end{abstract}

OCIS codes: $190.4970,320.7110$.

Optical parametric amplification (OPA) and optical parametric chirped-pulse amplification (OPCPA) have enabled the generation of intense, broadly tunable, few-cycle pulses for applications including ultrafast timeresolved spectroscopy, frequency metrology, and attosecond science [1,2]. The repetition rates of these systems have typically been in the $10 \mathrm{~Hz}-100 \mathrm{kHz}$ range due to speed constraints from pumping by solid-state regenerative and/or multipass amplifiers. Only recently, OPA systems with repetition rates in the $\mathrm{MHz}$ range have emerged with the help of $\mathrm{Yb}$-doped lasers based on fiber, thin-disk, and cryogenic cooling technologies with the design emphasis on the scaling of the average pump power [3-5]. Further scaling of the repetition rate in parametric amplifiers while maintaining peak intensity is highly desirable, because many applications can greatly benefit from the increased photon flux. As an alternative to average pump power scaling, enhancement cavities have been used to increase the usable peak intensity in a nonlinear process. Yanovsky and Wise demonstrated the frequency doubling of femtosecond Cr:forsterite oscillator pulses too weak for significant single-pass conversion [6]. Recently, Krishkek et al. used a UV enhancement cavity containing a BBO crystal to attain $7 \mathrm{~W}$ of intracavity UV radiation generating entangled photons via parametric downconversion at $81 \mathrm{MHz}$, which increased the detection sensitivity by 2 orders of magnitude [T]. Ilday and Kärtner proposed subharmonic cavityenhanced OPCPA (C-OPCPA), in which pump pulses are built up in a passive, high-finesse enhancement cavity containing a nonlinear crystal, seeded at a subharmonic of the pump repetition rate [8].

Recently, we proposed C-OPCPA with the full laser repetition rate [9]. In our scheme, pump pulses are coherently combined in a low finesse enhancement cavity transparent to signal and idler containing an OPA crystal in which a synchronized signal beam is amplified. We showed that if the pump and seed are sufficiently chirped, the cavity passively shapes the intracavity pump profile to attain more optimal conversion and increased bandwidth (BW), overcoming limitations set by the bellshaped pump intensity profile and the time-varying wavevector mismatch of the interacting pulses. Our numerical analysis predicted octave-spanning gain $\mathrm{BW}$ at high conversion efficiency and high repetition rate using the COPCPA with low average pump power sources [9]. This technique has the potential to amplify efficiently fewcycle sources without the need for pump power scaling. The relation between intracavity loss, $L$, and the output coupling value, $T$, determines system performance. When $L$, consisting of both a linear contribution and a nonlinear time-varying component from the intracavity conversion process, equals $T$ at all temporal coordinates, the cavity has achieved perfect impedance matching (IM), and therefore the reflected power vanishes and the enhancement factor, i.e., the ratio between the intracavity power and input power, becomes $1 / T$. At IM, all incident pump power is dissipated in the loss element, the nonlinear loss is maximized, and conversion is optimal. Reaching a condition closest to IM is the main issue for maximum conversion in C-OPCPA.

In this Letter, we present the first (to our knowledge) experimental demonstration of C-OPCPA. We show more than $50 \%$ conversion efficiency of coupled pump light at a high repetition rate $(78 \mathrm{MHz})$. C-OPCPA enables significant parametric amplification under conditions where an equivalent amount of pump power used in a singlepass configuration would lead to negligible conversion. Through comparison with the single-pass case, we evaluate the underlying impedance-matched conditions and relate them to the C-OPCPA performance. Figure 1 illustrates the optical layout. A Yb-doped fiber laser (YDFL) centered at $1.03 \mu \mathrm{m}$ seeds the OPCPA pump chain, and an Er-doped fiber laser (EDFL) centered at $1.56 \mu \mathrm{m}$ serves

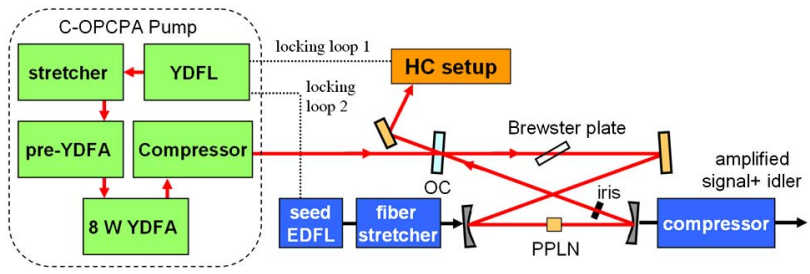

Fig. 1. (Color online) Schematic of C-OPCPA experiment YDFL/A, Yb-doped fiber laser/amplifier; EDFL, Er-doped fiber laser; HC setup, Hänsch-Couillaud locking scheme; OC, output/input coupler; PPLN, periodically poled lithium niobate. 
as the C-OPCPA signal. Both laser sources have a $78 \mathrm{MHz}$ repetition rate. The YDFL output is amplified in a conventional femtosecond chirped-pulse amplification system consisting of a grating stretcher, a home-built $\mathrm{Yb}$-doped fiber preamplifier, a commercial $8 \mathrm{~W}$ Yb-doped fiber amplifier, and a dielectric grating compressor. This pump system provides $5 \mathrm{~nm}$ in FWHM BW pump pulses with durations down to $500 \mathrm{fs}$ with up to $6 \mathrm{~W}$ average power. The EDFL signal pulses are stretched to $\sim 3$ ps using $5 \mathrm{~m}$ of single-mode fiber. The large seed pulse chirp and the chosen delay between pump and signal pulses optimized conversion efficiency for a well phase-matched $4 \mathrm{~nm}$ segment of the seed spectrum centered at $1565 \mathrm{~nm}$. This resulted in only $\sim 20 \mu \mathrm{W}$ of seed power overlapping the gain window set by the pump. The pump and stretched seed pulses are simultaneously injected into a $78 \mathrm{MHz}$ cavity with $10 \%$ output coupler, resonant with the pump pulses, and containing a 20-mm-long periodically poled lithium niobate (PPLN; HC Photonics, grating period $30 \mu \mathrm{m}$ ) crystal antireflection (AR) coated at the pump, signal, and idler wavelengths. We chose the PPLN because its large $d_{\text {eff }}$ value enables the use of low pulse energy at high repetition rate. Two telescopes were used to focus the pump and seed beams into the PPLN and match the closed cavity mode diameter of $100 \mu \mathrm{m}$.

The cavity was locked to the YDFL via the HänschCouillaud scheme [10]. The pump and signal source repetition rates were electronically locked [I1] by feedback to a piezo-mounted cavity mirror in the EDFL. We employed slow and fast feedback loops at $78 \mathrm{MHz}$ and $9 \mathrm{GHz}$, respectively, for high-precision locking. Less than $50 \mathrm{fs}$ of timing jitter was measured between the two lasers, which is sufficient considering pulse durations of $500 \mathrm{fs}$ and $3 \mathrm{ps}$ for the pump and signal, respectively. We first performed single-pass parametric amplification using the same C-OPCPA setup of Fig. 1 1 without closing the enhancement cavity, i.e., the output coupler was removed. Figure 2(a) shows the amplified signal power and the conversion to signal as a function of pump power, and Fig. 2(b)] shows the seed (dashed curve) and amplified signal spectra [blue solid (upper) curve] when $4 \mathrm{~W}$ of pump power were used. With $4 \mathrm{~W}, 500 \mathrm{fs}$ pump pulses and $\sim 20 \mu \mathrm{W}$ of seed power, we converted $5 \%$ of the pump power into signal at $78 \mathrm{MHz}$. Accounting for the idler at $3.03 \mu \mathrm{m}$, which was fully absorbed in BK7 collimating lenses, we estimate a total pump depletion of $\sim 8 \%$. Thus, with the same $4 \mathrm{~W}$ of intracavity power, the nonlinear loss in the closed cavity is expected to be $\sim 8 \%$ as well.
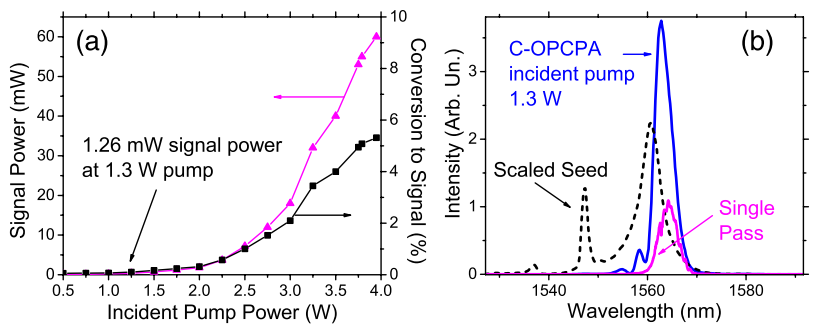

Fig. 2. (Color online) (a) Conversion to signal (black squares) and amplified signal intensity (magenta triangles) for the signal pass case; (b) signal input spectrum (black dashed), signal amplified via single pass (magenta), and signal amplified via C-OPCPA (blue).
If we allow for $\sim 2 \%$ linear losses from components in the cavity, i.e., mirrors and AR coatings on the PPLN, then the total intracavity loss can thus be tuned to $\sim 10 \%$. Thus, a $10 \%$ output coupler is suitable for IM. Additionally, the single-pass experiment shows that sufficient amplification can be achieved before crystal damage occurs at the desired power levels.

The performance of the C-OPCPA with $10 \%$ output coupling for IM was evaluated by monitoring the intracavity power, the reflected power, and the signal power under various seeded and unseeded scenarios. The first parameter evaluated was the coupling efficiency (CE). Higherorder modes are completely rejected by the cavity when locked on the fundamental mode. This uncoupled light does not contribute to conversion. The total conversion efficiency to signal and idler is therefore scaled by the CE. In principle, $\mathrm{CE}$ can be improved to be close to $100 \%$ with accurate spatial control of the input beam. To evaluate $\mathrm{CE}$, we adjusted the intracavity linear loss while monitoring the reflected and intracavity powers with low incident pump power. The linear loss was adjusted via aperture loss through an intracavity iris (see Fig. (I), which did not significantly disturb the cavity mode because the maximum introduced loss matched the output coupling value of only $10 \%$. As the intracavity linear loss was increased, the reflected power passed through a minimum while the enhancement monotonically decreased. At the minimum point of reflected power, the total loss equals the impedance-matched value and the enhancement experienced by the coupled light equals the inverse of the output coupling value. The reflected power at this value is the uncoupled amount, since otherwise it would vanish. The $\mathrm{CE}$ is therefore equal to 1 minus the ratio of the reflected power at this operating point to the incident pump power. We determine this to be $55 \%$.

Figure 3(a) shows, for the case in which no signal is present, the intracavity enhancement, the conversion to superfluorescence, and the percent reflected power. An enhancement of $\sim 30$ with a reflection of $\sim 70 \%$

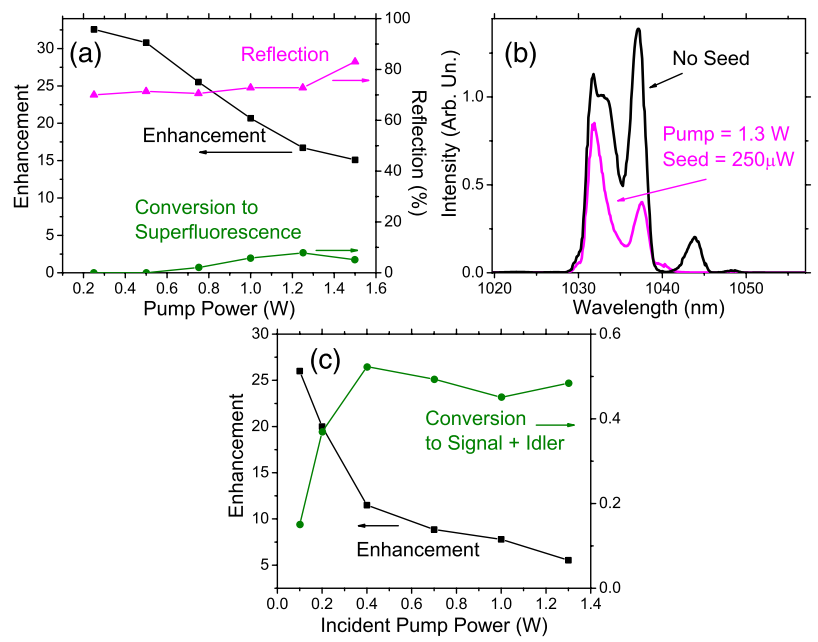

Fig. 3. (Color online) (a) Unseeded C-OPCPA: enhancement (black squares), reflection (magenta triangles), and conversion to superfluorescence (green circles). (b) Intracavity pump spectra for seeded (magenta) and unseeded (black) C-OPCPA. (c) Seeded C-OPCPA: enhancement (black squares) and total conversion (green circles). 
accounting for $\mathrm{CE}$ is recorded, consistent with an intracavity linear loss of 2\%-3\% (see Eq. (1) in Ref. [9]). Therefore, as stated earlier, a nonlinear loss via conversion to signal and idler of $7 \%-8 \%$ will give a total loss matching the output coupler and allow IM. At the highest incident pump power of $1.3 \mathrm{~W}$, the distortion of the intracavity power spectrum [Fig. 3(b)] is primarily due to conversion to superfluorescence but is also suggestive of slight selfphase modulation. Turning to seeded operation of the COPCPA apparatus, with only $1.3 \mathrm{~W}$ of incident pump power, $210 \mathrm{~mW}$ of the pump power is depleted into the signal, and we estimate $105 \mathrm{~mW}$ is depleted into the idler. The resulting signal spectrum is shown by the red solid (lower) curve in Fig. 2(b). In contrast, only $1.3 \mathrm{~mW}$ of amplified signal is observed in the single-pass configuration with this pump power [see Fig. 2(a)], which is 2 orders of magnitude lower. Allowing for $55 \% \mathrm{CE}$, with an effective available pump power of only $650 \mathrm{~mW}$ we are able to achieve nearly $45 \%$ pump depletion for a $78 \mathrm{MHz}$ source. As discussed below, even though the intracavity conversion is much less than $45 \%$, cavity enhancement with good average IM enables the dramatic conversion efficiency improvement with respect to the coupled incident pump.

For quantitative evaluation of the C-OPCPA performance and the underlying impedance-matched mechanism, we measured the total conversion to signal and idler and the enhancement versus incident pump power (including uncoupled light), as shown in Fig. 3(c). This figure reveals several features of the high repetition rate C-OPCPA. First, this figure demonstrates the cavity's ability to passively maintain high conversion in response to factors that reduce conversion. As the pump power increases from zero, the net conversion efficiency quickly increases and saturates at $\sim 50 \%$ for $>0.4 \mathrm{~W}$. Accordingly, the enhancement is first high $(\sim 26)$ and then quickly reduces to $\sim 10$ or less for $>0.4 \mathrm{~W}$. The cavity naturally employs increased enhancement to offset low incident power, thus maintaining intracavity peak intensity and conversion over a wide range of incident pump power. The passive self-adjustment results in the incident power threshold for amplification being $\sim 5$ times less compared to that of the single-pass amplification. Second, this curve allows us to characterize the impedance-matched operating point of the C-OPCPA system. Conversion of $>50 \%$ is observed between 0.4 and $1 \mathrm{~W}$ of incident pump power, where the enhancement ranges from 11.4 to 7.4 . The intracavity power spans $2.2 \mathrm{~W}$ to $4 \mathrm{~W}$. The variation in enhancement across this range is low and close to 10 , which is consistent with IM with a $10 \%$ output coupler. According to the single-pass measurements with $4 \mathrm{~W}$ of pump power, the total conversion is $\sim 8 \%$. Thus, the nonlinear loss in the cavity at this power is also $\sim 8 \%$. Additionally, the linear loss is $\sim 2 \%$, allowing the total intracavity loss to match the impedance-matched value of $10 \%$. The maximum C-OPCPA conversion of $55 \%$, however, is observed at $0.4 \mathrm{~W}$ of incident pump power. Accounting for $\mathrm{CE}$, the intracavity power at this operating point is $2.2 \mathrm{~W}$, significantly lower than the value predicted in the single-pass case. The discrepancy possibly comes from the mode mismatch between a single-pass pump beam and the cavity mode. A slightly larger beam size at the PPLN for the single-pass case would shift the single-pass conversion curve to high- er pump powers. Nevertheless, the trend observed in Figs. 3(c) and 2(a) is quantitatively consistent with the analysis for IM.

The advantage of using a cavity stems from increased effective nonlinear drive by recycling the pump power. The incident power required to achieve the desired intracavity pump power is reduced by the impedancematched enhancement factor, which is 10 in this case. Thus, under IM, the enhancement factor for the intracavity process significantly reduces the pump average power requirements. Additionally, only a fraction of the intracavity power is converted and is subsequently replaced by the next injected incident pump pulse. Thus a small fractional conversion of the intracavity pump power results in a large conversion of the incident pump power. In the absence of linear losses for a 10\% OC as in this case, an intracavity pump power level that allows a $10 \%$ singlepass total conversion yields $100 \%$ conversion of coupled pump light in the C-OPCPA case. With $3 \%$ linear loss, ideally $70 \%$ conversion is achievable (30\% into linear losses). Here, we have shown $55 \%$ conversion with only $400 \mathrm{~mW}$ of incident pump power. Nonlinear and dispersive effects play a role in limiting conversion, as does temporally nonuniform IM due to nonuniform pulse intensity profiles, but further improvements are expected as the cavity losses are reduced. Finally, we note that the pulse selfshaping mechanism predicted in [9], allowing BW enhancement, is impeded in this work by group-velocity walk-off effects. By appropriately adjusting system parameters, we expect to demonstrate $\mathrm{BW}$ improvement in future work.

In conclusion, we have demonstrated, for the first time, a significant conversion efficiency improvement in OPCPA by use of an enhancement cavity. This technique can maintain high conversion as the repetition rates in OPA systems are further scaled.

This work was supported by Air Force Office of Scientific Research (AFOSR) grants FA9550-08-1-0409 and FA9550-10-1-0063 and National Science Foundation (NSF) grant ECCS-1002286.

\section{References}

1. F. X. Kärtner, Few-Cycle Laser Pulse Generation and Its Applications, Vol. 95 of Topics in Applied Physics (Springer, 2004).

2. G. Cerullo and S. De Silvestri, Rev. Sci. Instrum. 74, 1 (2003).

3. T. Eidam, S. Hanf, E. Seise, T. V. Andersen, T. Gabler, C. Wirth, T. Schreiber, J. Limpert, and A. Tünnermann, Opt. Lett. 35, 94 (2010).

4. E. Innerhofer, T. Südmeyer, F. Brunner, R. Häring, A. Aschwanden, R. Paschotta, C. Hönninger, M. Kumkar, and U. Keller, Opt. Lett. 28, 367 (2003).

5. K.-H. Hong, A. Siddiqui, J. Moses, J. Gopinath, J. Hybl, F. Ö. Ilday, T. Y. Fan, and F. X. Kärtner, Opt. Lett. 33, 2473 (2008).

6. V. Yanovsky and F. W. Wise, Opt. Lett. 19, 1952 (1994).

7. R. Krischek, W. Wieczorek, A. Ozawal, N. Kiesel, P. Michelberger, T. Udem, and H. Weinfurter, Nat. Photon. 4, 170 (2010).

8. F. O. Ilday and F. X. Kärtner, Opt. Lett. 31, 637 (2006).

9. A. Siddiqui, J. Moses, K.-H. Hong, C.-J. Lai, and F. X. Kärtner, Opt. Lett. 35, 1929 (2010).

10. T. W. Hänsch and B. Couillaud, Opt. Commun. 35, 441 (1980).

11. D. J. Jones, E. O. Potma, J.-X. Cheng, B. Burfeindt, Y. Pang, J. Ye, and X. S. Xie, Rev. Sci. Instrum. 73, 2843 (2002). 